\title{
The Noise Monitoring System and Automatic Correction Algorithm
}

\author{
Zeng Huapu ${ }^{1}$, Li Yuting $^{1}$, Xu Huiyu ${ }^{1}, \mathrm{Pu}$ Yunming ${ }^{1 *}$, Qiao feng ${ }^{2}$ and Liang jun ${ }^{2}$ \\ ${ }^{1}$ School of Computer Engineering, Jimei University, Xiamen 361021, China \\ ${ }^{2}$ Xiamen Sunwe Science and Technology Co., Xiamen 361009, China \\ *yunmingpu@163.com
}

\begin{abstract}
For the improvement of living environment, there are more needs of the real time and online automatic noise monitoring system installed in many urban areas. In this project, the principle of noise audio frequency processing and coding algorithm was researched, including Discrete Cosine Transform and Wavelet Transform. The noise momitoring system solved the synchronous problem between the audio data and noise decibel, the special storage format separated the audio data which can be located in probability, and the audio data across segments to be played continally. The design of double-layer structure and isolating solved the problem of heat dissipating, waterproof and dustproof. In the experimental testing of the noise monitoring system, the noise decibel was affected by temperature and differential pressure, for improving its precision, the linear regression model is applied in the calculation of noise decibel The least estimate method was used to calculate the residual for the automatic porrection of the noise monitoring, and increase the accuracy of noise monitoring.
\end{abstract}

Keywords: environment nois inonitoring, remote monitor and control, linear regression, automatic correction, least estimates method

\section{Introduction}

With the rapid increment of economy, Chinese people enjoy the rich material life, but at the same time, people bear the cose of environmental degradation. In cities, especially in big cities, peoples want to breathe fresh air, drink clean water, sleep soundly, is becoming an extravagant needs. According to city environment situation survey, noise is a big effect on people' life, working and learning, especially the noise with more than $50 \mathrm{~dB}(\mathrm{~A})$ which decrease the quality of people's lives, leading to a variety of symptoms, such as cardiovascular diseases, memory loss and nervous breakdowns etc, which can seriously affect people's health [1]. Government now is paying more attention to the environment protection, and more money has been invested on environment pollution control.

Noise monitoring methods, monitoring density and monitoring frequency cannot simultaneously reflect city environment situation, with the expansion of the city, the density of building increased, the sound environment becomes more complex, although great efforts have been done by the environmental protection department, but it is still far from the peaceful living environment that residents persistent.

The continuous and automatic noise monitoring is necessary and possible for the environment protection. The research of the online noise monitoring system is important and necessary for the national economic development and people life quality, improve the noise environment and resident living need [2]. It is important for the construction of environment harmonious society too.

* Corresponding Author 
International Journal of Multimedia and Ubiquitous Engineering

Vol.11, No.9 (2016)

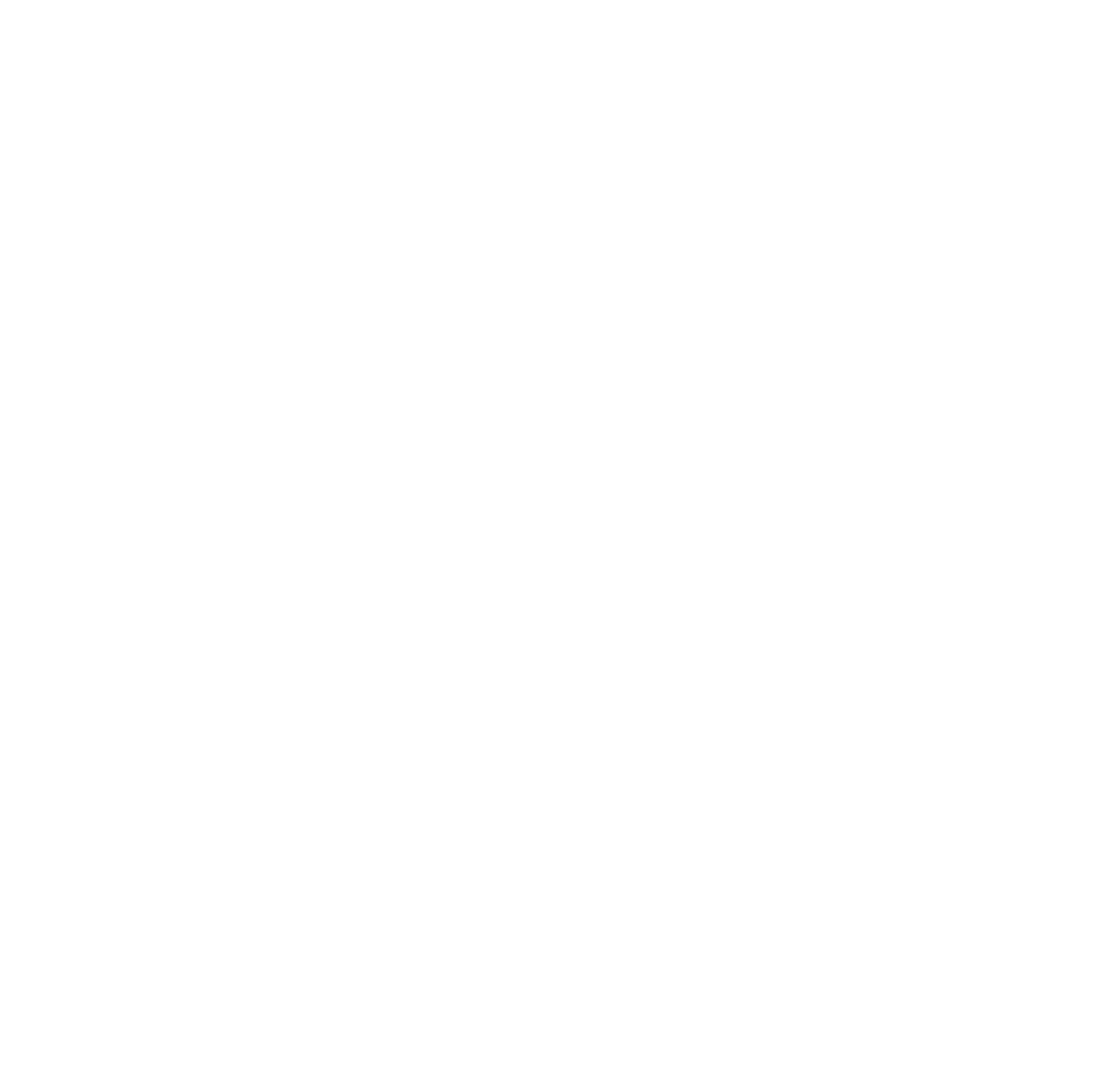




\section{Noise Monitoring Technology}

In abroad, environment noise monitoring equipment is able to measure, process data and transmit information automatically, and share monitoring information through network. Using computer to generate data map of the city noise condition, for the assessment of noise pollution and the urban design. As the technology improvement, miniaturization of noise measurement instruments have been developed, after years of development and modification, sound level meter from analog to digital, the function also from measures instantaneous sound level to measures equivalent continuous sound level, pulse sound level LI, cumulative percentile sound level LN so on.

As the environmental noise monitoring is using different methods depending on the measuring content. For the road traffic noise, using the natural road to distribute points, for regional environmental noise, using equal-area grid method to distribute points uniformly in the measurement area, for functional areas routinely, in each function area, arrange measuring point in the position where the functional characteristics is significant, and the position is representative, using a wireless network (WSN) for real-tine-and dynamic monitoring.

In terms of the method of evaluation, the most common is the weighted equivalent continuous sound level, which is commonly referred to equivalent sound level, with cumulative percentage sound level LN and circadian equivalent sound level Ldn in evaluation of the area of environmental noise, with traffic noise index TNI and other parameters in road traffic noise assessment. In evaluation of aviation noise monitoring, the weighted equivalent continuous perceived noise level(WECPNL) is used for evaluation [3].

In terms of the noise monitoring technology, the research work is focused on three aspects: first, predict environmental Cioise further more to provide the basis for environmental noise management and governance, control and planning; second, strengthen laboratory analysis of noise information, emphasize research on the information frequency components of noise time characteristics, spatial characteristics, and analog of noise information; and the third, improve the noise monitoring automatically, increase meniloring period, improve the monitoring data in time and space.

\subsection{The Principle of Noise Assessment}

In GB3096-2008, the Chinese acoustic environmental quality standards, the environmental noise of utban was divided into five areas, see Table 1.

Table 1. Urban Area Noise Standards (Db)

\begin{tabular}{c|c|c|c}
\hline No. & Area & Daytime & Night \\
\hline 1 & Wellness, superior Villa, superior Hotel Zone & 50 & 40 \\
\hline 2 & $\begin{array}{c}\text { Living, cultural and educational institutions dominated } \\
\text { area }\end{array}$ & 55 & 45 \\
\hline 3 & Living, commercial, industrial mixed zone & 60 & 50 \\
\hline 4 & Industrial Area & 65 & 55 \\
\hline 5 & $\begin{array}{c}\text { Urban roads, transportation routes, inland waterways, } \\
\text { both the sides of the area of railway. }\end{array}$ & 70 & 55 \\
\hline
\end{tabular}

According to national standards, residential noise during the day cannot exceed $50 \mathrm{~dB}$, and less than $45 \mathrm{~dB}$ at night, if higher than this standard, it will be harm to humans. In "GB22337-2008 social life environmental noise emission standards", bedroom noise limits: daytime $40 \mathrm{~dB}$, night $30 \mathrm{~dB}$, others noise limits: daytime $45 \mathrm{~dB}$, night $35 \mathrm{~dB}$. In measuring instruments, for different frequencies objective sound pressure levels artificially given appropriate increase or decrease, this correction method is called frequency weighting, the frequency weighting networks in noise meter are mainly three 
standard weightings A, B and C. A weighted simulate the human ear's response of equal loudness curve 40 square pure tones, it's noise meter curve shape is opposite with equal loudness curve of 340 square, thus the middle and low band have a large attenuation. B weighted simulate the human ear's response of 70 square pure tones, thus low band has certain attenuation. $\mathrm{C}$ weighted simulate the human ear's response of 100 square pure tones, over the entire audio frequency range have nearly flat response. Sound Level Meter through frequency weighting network measured sound pressure levels is called the sound level, according to the weighted nets used, the sound level is divided into A, B and C sound level [4].

A sound level reflects the relationship between noise and frequency, for steady noise, that noise is little change over time, we can usually use A sound level to evaluate. Equivalent sound level is the amount of non-stationary noise evaluation which based on A sound level, at the same exposure time be able to give an equal number of sound energy, the sound level is change noise's equivalent sound level, also known as the equivalent A sound level, or simply called equivalent sound level. Equivalent continuous A sound level is A sound level which unsteady noise within a certain period of time, energy a eraging method, a continuous unchanged of A sound level is used to indicate the no se sound level in the period, also known as energy A sound level, equivalent continuous sound level Leq calculated according to formula (2-1):

$$
L_{e q}=10 \lg \left(\frac{1}{T} \int_{0}^{t} 10^{L_{A} / 10} d t\right.
$$

$\mathrm{L}_{\mathrm{A}}$ is the instantaneous $\mathrm{A}$ sound level of time $\mathrm{l}, \mathrm{T}$ is the total time of continuous measurement.

Cumulative percentile sound level is an indicator used to evaluate the statistical distribution of the noise intensity time in measurement period, is minimum of A sound level within cumulative time. The most comnonly used is the L10, L50, L90. L10: Within measuring time, more thar $0 \%$ of the noise level, equivalent to the average peak of noise. L50: within measuring time, more than $50 \%$ of the noise level, equivalent to the average of noise. L90: within measuring time, more than $90 \%$ of the noise level, equivalent to the value of the background of noise [5].

Day and night equivalent sound level Ldn, is used to indicate changes in the noise on day and night, night hoise increase $10 \mathrm{db}$ weighted. Since the use of day and night equivalent sound, achieved great success, the number of residents affected by noise has a good correlation with day and night equivalent sound level.

$$
L_{d n}=10 \lg \left[\frac{1}{24}\left(\mathrm{~T}_{d} * 10^{0.1 * L_{d}}+\mathrm{T}_{n} * 10^{0.1 *\left(\mathrm{~L}_{n}+10\right)}\right]\right.
$$

Ldn is the day and night equivalent sound level, dB (A), Ld is daytime noise level, $\mathrm{Ln}$ is night noise level, $\mathrm{Td}$ is daytime noise exposure time (hours), $\mathrm{Tn}$ is night noise exposure time.

Sound fevel meter was used in noise measurement, the meter response can be divided into four categories according to the sensitivity:

1. Slow, Header time constant $1000 \mathrm{~ms}$, generally used to measure steady noise, the measured value is a valid value.

(2) Fast, Header time constant $125 \mathrm{~ms}$, generally used to measure unsteady noise and transportation noise. The fast speed is close to the human ear responds to sound.

(3) Pulse or pulse hold, handles rise time $35 \mathrm{~ms}$, for measuring the pulse noise which has longer duration, the measured value is the maximum valid value.

(4) Peak hold, gauge needles rise time less than $20 \mathrm{~ms}$, was used to measure pulse sound which has a short duration. The measured value is peak value which is the maximum value. 


\subsection{The Principle of Noise Audio Processing}

Noise monitoring system must first complete digital audio processing, it generally has three steps, the sampling, quantification and encoding. Computer sampling digits generally has eight bits or 16 bits, the sampling frequency generally has $11 \mathrm{KHz}, 22 \mathrm{KHz}$, $44 \mathrm{KHz}$. The sampling is a sound signal which continuous changing from the time to intercept several sample values, the quantization is the rounding processing of amplitude value of sampled audio signal values, which use the uniform quantization and nonuniform quantization scheme. Coding is to convert the signal into a digital pulse code after sampling and quantization. Audio coding technology is generally divided into: waveform coding, parameters coding and hybrid coding [6].

Firstly, the waveform coding is to convert a time-domain signal into a digital code directly, trying to make the speech waveform of the reconstruction maintain the shape of the speech waveform of the original. The basic principle of waveform coding is an analog voice on a time axis to sample by a certain rate, then the amplitude of the sample will be quantified through layered and coded. Decoding is the reverse process of it, decoding and filter the received digital sequence back into an analog signal. Pulse code modulation (PCM), differential DPCM and incremental modulation (DM), and their various modified adaptive delta modulation (ADPCM), adaptive differential encoding (ADPCM), etc., are all belong to waveform coding techniques. The transmitting rate is $64 \mathrm{Kbps}$ or $16 \mathrm{Kbps}$, can give high coding quality, and if the rates decline further, its performance will decline rapidly [7].

Secondly, the parameter coding, also known as a sound-source code, extracting the signal characteristic parameters which the source signal in a frequency domain or other orthogonal transform domain, converting into digital code for transferring. At the decode end, trying to reconstruct the original seech signal, the ompression rate is high. But the algorithm complexity, the naturalness of synthesiked speech is not good, anti-noise ability is poor. A typical parametric enceder has formant vocoder, homomorphic coding and linear prediction vocoder. The compression ratio is large, cheap, but the large amount of calculation, quality is not high, and sensitive to the environmental noise.

Thirdly, the hybrid coding use more than two kinds of coding method simultaneously, if combined with the waveform coding and parameter coding method, can gathered both advantages of the code, such as MPLPC, KPELPC, and CELP etc.

Audio coding algorithm generally using block transform processing method, such as the discrete cosine transform (DCT), wavelet transform. Because the audio coding algorithm based on block transform is useful for removing the signal redundancy, improve the compression ratio, Audio coding algorithm based on the strong correlation between channels, throught the audio signal has transmitted and related additional information to reconstruct audio signal, audio transform is convert time domain into the frequency domain transform [8].

\subsection{One-Dimensional Discrete Cosine Transform}

Definition: $\{x(m) \mid m=0,1, \ldots N-1\}$ is a sequence of data of bandwidth limited signal sampling, there are $\mathrm{N}$ sampling values, defined as a one-dimensional discrete function, and the positive transform definition:

$$
\begin{gathered}
\mathrm{Y}(0)=\frac{1}{\sqrt{N}} \sum_{m=0}^{N-1} x(\mathrm{~m}), \quad \mathrm{k}=0 \\
\mathrm{Y}(\mathrm{k})=\sqrt{\frac{2}{N} \sum_{m=0}^{N-1} x(\mathrm{~m}) \cos \frac{(2 m+1) \mathrm{k} \pi}{2 N}, \mathrm{k}=1,2, . ., \mathrm{N}-1}
\end{gathered}
$$

And the inverse transform definition:

$$
\mathrm{x}(\mathrm{m})=\frac{1}{\sqrt{N}} Y(0)+\sqrt{\frac{2}{N}} \sum_{k=1}^{N-1} \mathrm{Y}(\mathrm{k}) \cos \frac{(2 \mathrm{~m}+1) \mathrm{k} \pi}{2 N}, m=0,1, \ldots n-1
$$


The audio source through the DCT transform achieve coefficient matrix of cosine transform:

$$
\mathrm{X}_{A}=[\mathrm{Y}(0), \mathrm{Y}(1), \ldots \mathrm{Y}(\mathrm{N}-1)]^{\mathrm{T}}
$$

Through the DCT transform, the audio signal source not only remove the correlation between the audio signal source and $\mathrm{Y}$, but also make the audio signal source energy concentrate in a few transform coefficients of $\mathrm{Y}(0), \mathrm{Y}(1), \ldots, \mathrm{Y}(\mathrm{N}-1))$, these coefficients compose several coefficient tape, the benefits is that the coefficients tape can code and decode separately. Professor Gou designed the variable step size LMS algorithm to adapt noise cancellation based on DCT transform, in convergence speed, steady-state offset and algorithm complexity aspects have achieved good results [9].

\subsection{Wavelet Transform}

The basic principle of the wavelet transform comes from stretching and translation, with the traditional Fourier transform by trigonometric expansion compared, wavelet function is fast decay, fully smooth, energy compact and mutual generate function collection by stretching and translation.

Wavelet algorithms is a fast algorithm which generally firstly transform the data in the horizontal direction, and then transform the data in the vertical direction, it is a transformation method which has time-frequency localization, which is effective to characterize the non-stationary signal of audio in the frequency domain. Wavelet transform improve the performance of the codec, the audio signal source made three discrete wavelet transform, the audio transform coefficients-are divided into coefficient blocks, the coefficient blocks are divided into the block hopping, the intra block and inter block, then coding the low frequency coêfficients in LDPC codec.

Wavelet Based on this consideration for the processing of the signal source, highfrequency spectral information, the fime interval should be relatively small to give a high accuracy, for the low-frequency spectral information, the time interval is relatively wide in order to give a complete information, that there is a flexible variable time frequency window, when at high center frequency is automatically narrowed, widened automatically at low center frequency [0]. Wavelet transform definition:

The transform kernel function

$$
W f(\mathrm{a}, \mathrm{b})=\int f(\mathrm{t}) \psi_{a, b}^{*}(\mathrm{t}) \mathrm{dt}
$$

$$
\circlearrowleft \Psi_{a}(\mathrm{t})=\frac{1}{\sqrt{a}} \psi\left(\frac{t-b}{a}\right), \mathrm{a}>0, \mathrm{~b} \in \mathrm{R},
$$

$\psi(\mathrm{t})$ is called basic wavelet or mother wavelet, it is generally a band-pass function when $t=0$ as the center in the time domain, the time domain or frequency domain must be localized (compact support), and the average value is zero, that is $\int \psi(\mathrm{t}) \mathrm{dt}=0$.

\section{ENS Noise Monitoring System}

Noise online monitoring system can monitor the sound environment 24 hours a day, find out the strength, type and regular of generation of excessive noise immediately, and the law enforcement can enter the scene to collect noise evidence immediately and accurately. The punishment can be carried out for the excessive noise pollution. Faced with tough pollution of sound environment, traditional artificial monitoring could not meet the needs of automation, network and intelligence. For the monitoring of the sound environment immediately, accurately in full scale, and supervise the source of pollution, the noise online monitoring system is quite important and necessary. 


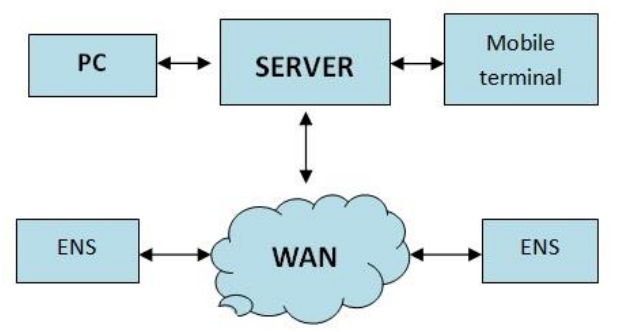

Figure 1. Noise Monitoring System

The online monitoring system is based on the unit of noise environment monitoring unit which was installed in different areas. It can transmit the noise data to the center of environment monitoring immediately according to the monitoring needs. In the center, the law enforcement can not only know the status of sound environment of the whole city, but also know the exact figures and its type. Provide powerful evidence of noise pollution. Moreover, the analysis and modeling in big data of sound environment witl favor the decision of management fully, and provide a basis of creating environmental legislation and creating the standard of environment noise. Protect the health of people and their hearing, sleep and thinking etc.

ENS-3000 is an online monitoring product of noise which is integration, full-featured, practical, and stability is high. The product has the only one power source which is high integrated, and has reliable performance in outdoor settings. Figure 1 is the structure of noise monitoring system.

The online monitoring system is modular structure which is a configurable and definable, the communication of the confrgurable modules use message queue process. Modules are free-running executable files; different module could exist in the system as free-running process the system is running. Modules communicate in the same way of processes, and the communicate relationshipbetween modules could be configured by INI file. Without changing other modules, replacing the executable file of one module will update the module, modify the INI file will update the communication relationship. Delete the executable file of one module from the disk will disable the module, and it won't have an effect on the performance of other modules.

\subsection{Decibe Data Acquisition Module}

Noise decibel collection read ten original noise decibel from the sound-level monitor per second, calculate out the equivalent Leq and the maximum Lmax per second, and transmit the Leq Lmax per second and original noise decibel(optional) by message queue. This module deal with the event of sound calibration, during the run time, the noise decibel is shielded. Data collecting use the equivalent distance and equivalent continuous 1 sound level calculation. Because of the range of frequencies and sound $20 \mathrm{~Hz} 20000 \mathrm{~Hz}$, in the algorithm, the frequencies and sound is divided into several successive bands, and set a band pass filter for every band to test the sound pressure. The sound interval of bands of two frequencies which the frequency ratio is $2^{1 / n}$. The sound interval is always $1 / 1$ and $1 / 3$. Every sound interval is named by its center frequency $f_{0}$, upper cut-off frequency $\mathrm{f}_{\mathrm{u}}$, lower cut-off frequency $\mathrm{f}_{1}$ and bandwidth $\mathrm{B}$.

$$
\begin{gathered}
f_{0}=\sqrt{f_{u} f_{1}} \\
f_{1}=2^{-\frac{1}{n}} f_{u}=2^{-\frac{1}{2 n}} f_{0} \\
f_{u}=2^{\frac{1}{n}} f_{1}=2^{\frac{1}{2 n}} f_{0} \\
B=f_{u}-f_{1}=\left(2^{\frac{1}{2 n}}-2^{-\frac{1}{2 n}}\right) f_{0}
\end{gathered}
$$




\subsection{Audio Data Acquisition Module}

Audio data collection read 16bit audio collection data 1575 times(3150 bytes) from sound card, $11 \mathrm{KHz}$ audio reading 7 times per second, $22 \mathrm{KHz}$ audio 14 times and $44 \mathrm{KHz}$ 28 times. After reading 1575 16bit audio collecting from sound card, it will transmit the audio data to the relative module. Audio data deal with the event of sound calibration, during the run time the audio data collected will be shielded. To simplify the program, the sound card is working all the time.

\subsection{Excessive Noise Handling and Transmission}

This module is used to compress the audio data in OGG audio compression technology, divide the audio into 5 minutes logic section, judge excessive event according to the threshold of collection rule. After collecting the audio data and decibel data 5 minutes(1 logic time), this module will push the data into buffer, and the data transmission will send the data from buffer to the server.

\subsection{Real-Time Data Handling and Transmission}

This module is used to deal and transmit the real-time decibel data and real-time audio data. This module will monitor and decide whether to deat the real-time data according to the "real-time data collection" which is send from serve

\subsection{Statistics Calculation and Transmission}

This module is used to calculate 10 ninutes statistics, One-hour statistics, 24-hour statistics, and push the data into buffer, then the data ransmission will send the data from buffer to the server.

\subsection{Other Module}

Other modules includes watchdog of software, controlling LED, controlling LCD, handling LOG and transmission, handling debug information and transmission, online upgrade, time synchronize, system rûn time maintenance.

\section{The Key Technology of ENS}

(1)The technology of Coording successive audio data, in this technology, the successive audio data is unable to store in just one audio file, because by the increment of the audio data, it wil be difficult to store, compress and locate the audio data. To record the successive andio data, and locate any one point in time rapidly then get audio data of any length start form the point, the usable method is to store and compress the audio data segmentation for the solution of the problem mentioned before. And the problem of discontinuty of audio which is caused by the audio segment is solved too, come to the expectation.

(2) The technology of cooling, dustproof and waterproof, the design of closed structure is able to solve the problem of dustproof and waterproof, but it is useless to cool machine. So place the ventilation holes on the box is able to ensure good ventilation in the box, but it will come with the dustproof problem. After many testing, solving the problem of cooling the machine, dustproof, waterproof by the design of buffering and the two-tier cooling.

(3)The output technology of the noise decibel and audio data from one sensor, there are three reasons of using one sensor: simplify the input channel of signals; simplify the structure of hardware of this system; improve the stability and reliability. To make the sound level meter and sound card share the sound sensor, we add the relative circuit, the 
measures of shielding and isolating solve the problem of interference caused by the share of sensor.

\section{Automatic Correction Algorithm}

\subsection{Linear Regression Principal}

The principal of linear regression model is as following. And $\beta$ is partial regression coefficient, $\varepsilon$ is stochastic error.

$$
y_{i}=\beta_{0}+\beta_{1} x_{1}+\beta_{2} x_{2} \cdots+\beta_{p} x_{p}+\varepsilon_{i}
$$

The multivariable function is:

$$
[\mathrm{b}, \text { bint, } \mathrm{r}, \text { rint, stats }]=\operatorname{regress}(\mathrm{y}, \mathrm{x}, \mathrm{a}) .
$$

And $b$ is statistical probability estimation of $\beta, b=\{\beta 0, \beta 1, \beta 2 \ldots\}$; bint is the Interval Estimation of regression coefficient, $r$ is residual, rint is the Confidence interval of residual, stats: coefficient of determination $\mathrm{R} 2 、 \mathrm{~F}$ inspection value and probabiluty $\mathrm{R} 2$ is range from 0 to 1 , the most is better, and the least of probability is better [1-12

In experimental testing, the noise value is dependent on the environment factors, such as temperature, air pressure and humidity, after analyze deeply, the mainly factors are temperature and air pressure. So, the regression mode is as formula (5-2), y is noise monitoring value, $\mathrm{x} 1$ is temperature, $\mathrm{x} 2$ is air pressure. For the simply processing, ignore stochastic error $\varepsilon$.

$$
y_{i}=\beta_{0}+\beta_{1} x_{i_{1}}+\beta_{2} x_{2}
$$

The residual $\mathrm{e}_{\mathrm{i}}$ is the difference between observed value $Y_{1}$ and predicted value $\hat{Y}_{i}$ of $\mathrm{X}_{\mathrm{i}}$, according to the least square estimate principle, the estmation method makes the $\mathrm{e}_{\mathrm{i}}$ to achieve the minimum at all sample points, the error sum of squares $Q$ is minimum.

$$
Q=\sum_{i=1}^{n} e_{i}^{2}=\sum_{i=1}^{n}\left(y_{i}-\hat{y}_{i}\right)^{2}
$$

\subsection{The Experimentaldata Analysis}

The experimental data $M 1, M 1=\{x 1, x 2, y\}$, and $\mathrm{x} 1$ is temperature, $\mathrm{x} 2$ is differential pressure, and y is noise level. And M 1 is used to calculated in MATLAB.

$x 1[54]=27.2,26.7,26.5,26.8,27,27.60,27.50,27.90,27.80,26.90,27.30,27.4,28.5,29.1,28$ $.8,28.4,28.6,293,30.2,30.4 .30 .2,27.6,28.8,27.3,26.1,25.9,25.3,24.1,25.1,24.8,24.9,24.8,24$ $.6,24.7,25.8,25,24.6,25.2,26,29.5,30.4,30.5,30.1,30.4,29,29.1,31.1,30.8,31.4,31.6,27.8,27$. $7,28.3,30.5\}$

$\mathrm{x} 2[54]=\{-3.99,2.65,-2.05,-2.49,-2.9,-4.75,-5.25,-5.45,-5.25,-3.75,-3.35,-2.25,-3.25,-$ $4.75,-5.05,-3.95,-3.65,-3.25,-11.15,-11.45,-11.85,-11.25,-7.75,-6.95,-6.25,-2.15,-4.35,-$ $3.75,-4.15,-15,-4.05,-4.25,-4.65,-4.65,-7.05,-6.95,-6.15,-5.75,-10.75,-5.65,-6.45,-8.75,-$ $8.95,-8.95,-6.25,-8.25,-9.15,-10.75,-10.25,-10.55,-10.45,-9.95,-10.15,-6.25\}$

$y[54]=\{93.8,93.9,94 \cdot 1,94,93.9,93.70,93.70,93 \cdot 60,93.70,93.80,93.90,93.9,93.8,93.7,93$. 8.93, 7,93.8,93.8,93.7,93.7,93.8,93.6,93.8,93.6,93.7,93.7,93.7,93.6,93.7,93.8,93.9,93.8,93. $7,93.6,93.7,93.7,93.7,93.7,93.5,93.9,93.8,93.8,93.8,93.8,93.8,93.7,93.8,93.7,93.8,93.8,94$. $3,94.2,93.8,93.8\}$

Step1, M1 data used in linear regression model, and the residual plot is as Figure2: 


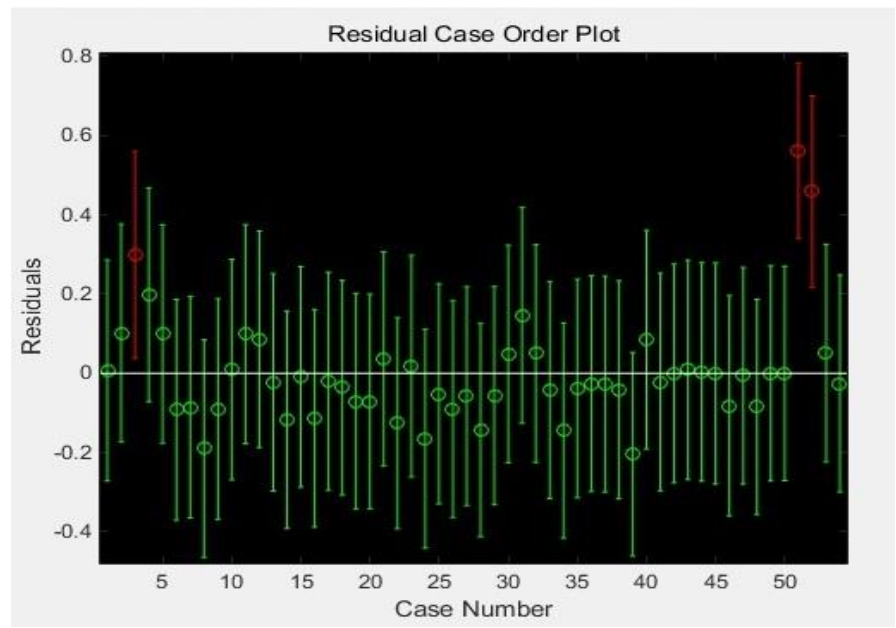

Figure 2. First Regression Residual Plot

$\mathrm{b}=\{93.3768,0.0168,0.0102\}$

bint $=\{(92.8155,93.9381),(-0.0050,0.0387),(-0.0056,0.0259)\}$

stats $=0.0502,1.3466,0.0195$

Step2, in Figure 2, residual of element 3,51,52 were distinctly deviate from other data, so delete these 3 elements from M1, get M2, and used in linear regression model, the residual plot is as Figure 3:

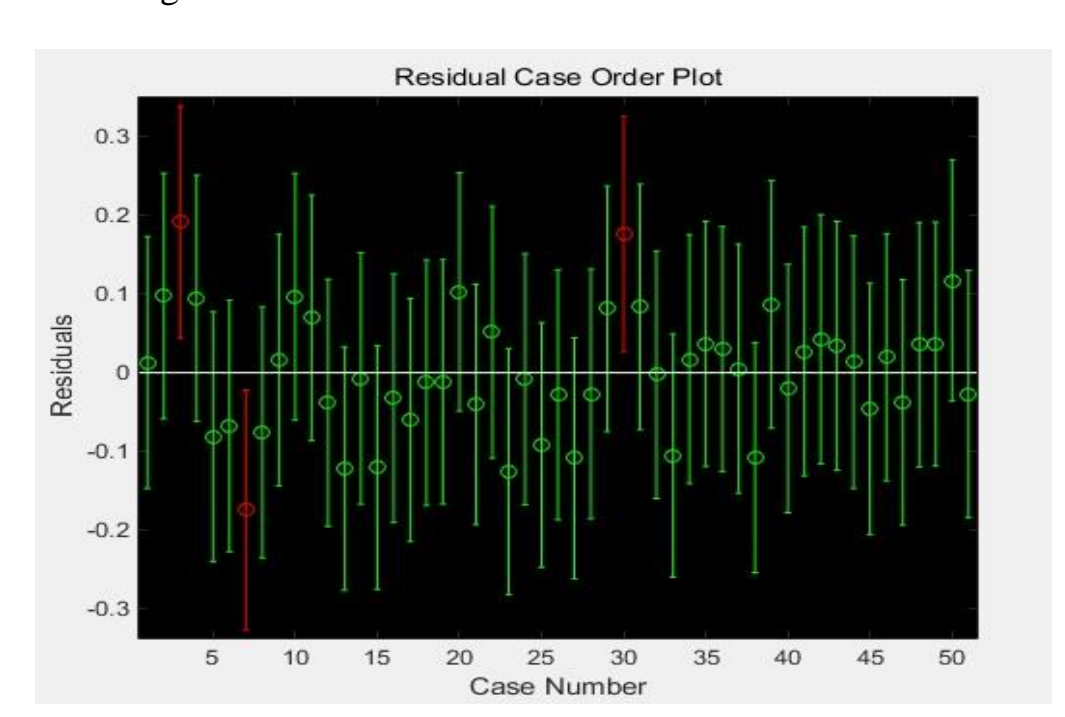

Figure 3. Secondly Regression Residual Plot

$\mathrm{b}=\{93.1391,0.0270,0.0217\}$

bint $=\{(92.8133,93.4650),(0.0142,0.0398),(0.0120,0.0314\}$

stats $=0.3402,12.3730,0.0064$

Step3, in Figure 3 residual of element 3,7,30 were distinctly deviate from other data, so delete these 3 elements from M2, get M3, used in linear regression model, and the residual plot is as Figure 4: 


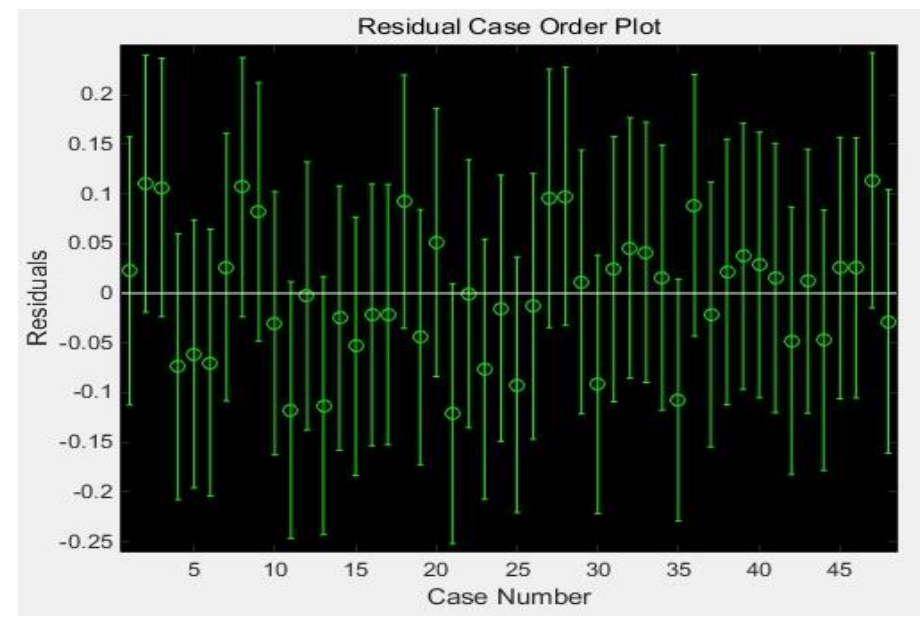

Figure 4. Thirdly Regression Residual Plot

$\mathrm{b}=\{93.0659,0.0291,0.0200\}$

bint $=\{(92.7856,93.3462),(0.0181,0.0400),(0.0116,0.0283)\}$

stats $=0.4289,16.8982,0.0046$

In the Figure4 residual plot, there is no element residual deviate from other data, is uniform distribution. The result of M3 is better than M1 and M2, such as the probability 0.0046 in step3 is better than 0.0064 in step2, and 0.0195 in step1, so the noise automatic correction formula is as formal (5-1). The corrective noise value is more accuracy and valuable.

\section{Conclusion}

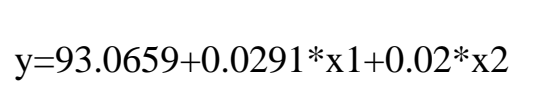

ENS noise monitoring system favors the use of long time, reliable real-time monitoring and alarming, saving datacontinuous play back. It provides scientific basis and decision for voice control, intelligent traffic environment protecting etc. As the experimental result, the linear regression algorithm applied in noise monitoring system, improve the accuracy of the rose monitoring

The application of ENS noise monitoring system, meets the needs of the society and economy development, achieve to improve the people's livelihoods, improve the sound environment of city, increase the consciousness of residents to take part in managing and maintain the silentliving environment.

\section{Acknowledgements}

The research work was supported by Natural Science Foundation of Fujian Provincial under Grant No. 2013J01243 and Science Project Foundation of Fujian Provincial under Grant No. 2013H0032.

\section{References}

[1] Q. Qin, Z. Bin and D. Chuanbo, "The Development of Environmental noise Wireless-Monitor System Research", Environmental Monitoring in China, vol. 23, no. 6, (2007), pp. 38-41.

[2] Y. Xusheng, Y. Jianghua and X. Yongsong, "Study on environmental noise remote monitor technology based on CDMA networks", Piezoelectrics \& Acoustooptics, vol. 34, no. 3, (2012), pp. 466-469.

[3] Z. Limin, L. Yadan and M. Yuying, "Research on Application of Minimum Distortion Point in Distribution of Noise Monitoring", Journal of Changchun University of Science and Technology(Natural Science Edition), vol. 34, no. 4, (2011), pp. 106-109. 
[4] C. Xiaohuan, Y. Jianhua and C. Liwei, "Design of Distributed City Noise Monitoring System Based on Wireless Sensor Network", Chinese Journal of Sensors and Actuators, vol. 26, no. 8, (2013), pp. 11591162.

[5] Y. Lei, T. Xian and M. Jian, "Online Metrological Study Oil Key Parameters of Environmental Noise Monitoring Terminals", Noise and Vibration Control, vol. 33, no. 4, (2013), pp. 232-236.

[6] Y. Jianxiong, "Design of Environmental Noise Monitor System Based on VI and Web", Jisuanji yu xiandaihua, vol. 1, no. 3, (2010), pp. 81-83.

[7] Y. Degu and Z. Shaoli, "The Research for Distributed Source Audio Coding Method", Journal of Jiangxi Normal University(natural science), vol. 35, no. 1, (2011), pp. 54-56.

[8] W. Hao, T. Xianghong, C. Hongbing and W. Yong, "DCT Domain Video Watermarking Algorithm Based on the Interval", Journal of Hangzhou Dianzi University, vol. 34, no. 2, (2014), pp. 20-23.

[9] G. Xinke, C. Zhuo and L. Dongdong, "Variable step-size LMS algorithm based on discrete cosine transforill for noise cancellation", Journal of Lanzhou University of Technology, vol. 40, no. 2, (2014), pp. 93-97.

[10] B. T. Ting, D. C. Xia and G. Ying, "Image Edge Detection Based on Wavelet Transform and Canny Operator", Journal of Harbin University of Science and Technology, vol. 15, (2010), pp. 44-51.

[11] L. Yuwu and F. Xiaozhao, "Kernel linear regression for face recognition", Neural Computing and Applications, vol. 24, (2014), pp. 1843-1849.

[12] Lai P.Y, Lee and M. S. Stephen, "Estimation of central shapes of error distributions in linear regression problems", Annals of the Institute of Statistical Mathematics, vol. 65, (2013), pp. 105-124. 


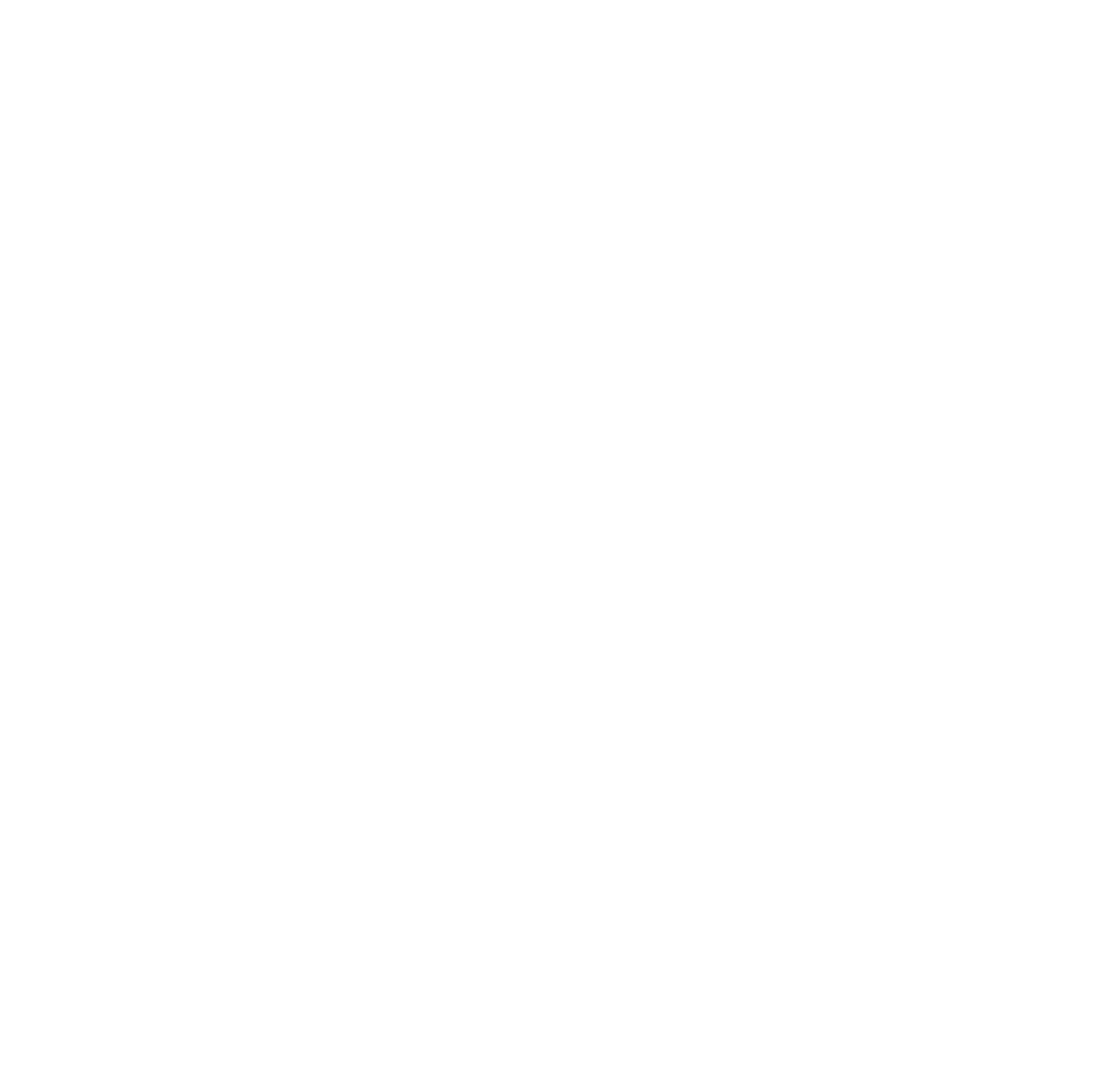

